\title{
The Effects of Postnatal Retinoic Acid Administration on Nephron Endowment in the Preterm Baboon Kidney
}

\author{
MEGAN R. SUTHERLAND, LINA GUBHAJU, BRADLEY A. YODER, MILDRED T. STAHLMAN, AND M. JANE BLACK \\ Department of Anatomy and Developmental Biology [M.R.S., L.G., M.J.B.], Monash University, Clayton, Victoria 3800, Australia; \\ Department of Pediatrics [B.A.Y.], University of Utah, Salt Lake City, UT 84158; and Department of Pediatrics [M.T.S.], Vanderbilt \\ University, Nashville, TN 37232
}

\begin{abstract}
Administration of retinoic acid (RA), the active metabolite of vitamin A, is linked to the stimulation of nephrogenesis. The aim of this study was to determine whether early postnatal administration of RA could enhance ongoing nephrogenesis in a baboon model of premature birth. Unbiased stereological methods were used to estimate kidney volume, renal corpuscle volume, and nephron number. The percentage of abnormal glomeruli and the number of glomerular generations was also determined in the kidneys of preterm control $(n=6)$ and preterm + RA $(n=6)$ animals that received $500 \mu \mathrm{g} / \mathrm{kg} / \mathrm{d}$ of all-trans RA after premature delivery. There was no significant difference between the preterm control and the preterm +RA groups in kidney size, nephron number (preterm control: 329,924 \pm 41,752; preterm +RA: 354,041 \pm 52,095; $p=0.59$ ), renal corpuscle volume, number of glomerular generations, or the percentage of abnormal glomeruli. The proportion of abnormal glomeruli did not appear to be linked to any elements of postnatal care examined. The results of this study indicate that early postnatal administration of RA is unable to stimulate nephrogenesis in the kidney of the preterm baboon. Encouragingly, it does not appear to have any adverse effects on kidney development. (Pediatr Res 65: 397-402, 2009)
\end{abstract}

A dvances in neonatal care have markedly improved the survival of preterm infants such that neonates as young as $26 \mathrm{wk}$ gestation (term $=37 \mathrm{wk}$ ) now have a $60-80 \%$ chance of survival $(1,2)$. There is limited knowledge to date, however, as to long-term adverse health consequences of preterm birth, and the possibility that these may be prevented through neonatal intervention.

A major organ that rapidly develops during late gestation is the kidney, with the formation of nephrons (nephrogenesis) continuing until approximately 36 wk gestation (3). Furthermore, $60 \%$ of nephrons are developed during the third trimester of pregnancy (4). Infants born preterm, therefore, are delivered before nephrogenesis is completed; it is conceivable that this will impact upon their future nephron endowment and kidney function. Indeed, the results of a recent study by Gubhaju et al. (Gubhaju L et al. The effects of preterm birth on nephrogenesis, 32nd Annual FNPS Meeting, September 25-28, 2005, Glenelg, Australia, Abstract O8), in a nonhuman

Received June 9, 2008; accepted November 4, 2008.

Correspondence: Megan Sutherland, BBiomed Sc, Department of Anatomy and Developmental Biology, Post Office Box 76, Monash University, Victoria 3800, Australia; e-mail: Megan.Sutherland@med.monash.edu.au

Supported by NIH HL52636 BPD Resource Center grant, P51RR13986 for facility support, the Vanderbilt Newborn Research Fund, and the National Health and Medical Research Council of Australia. primate model of preterm birth, has indicated that an attenuation of the nephrogenic potential of the individual may occur due to preterm delivery, suggesting that nephrogenesis in the extrauterine environment is impaired. In a human postmortem analysis, Rodriguez et al. (5) determined that, although nephrogenesis is ongoing after preterm birth, infants had a significantly reduced number of glomerular generations which is potentially indicative of a nephron deficit. Furthermore, a number of the preterm infants exhibited glomerular abnormalities, such as cystic dilation of the Bowman's space. This deficit in functional nephrons may in turn lead to long-term complications, as a reduced nephron endowment has been linked to an increased susceptibility to hypertension and the development of renal disease later in life (6-8). Therefore, it is essential that nephron number be maximized before the completion of nephrogenesis.

Retinoic acid (RA), the active metabolite of vitamin A, acts as a transcription factor and modulates the expression of the Ret tyrosine kinase receptor (9). Increased Ret signaling leads to an increase in ureteric bud branching in the developing kidney, and through this mechanism RA has been linked to the modulation of nephron number both in vitro (10) and in vivo. Importantly, a clear linear correlation has been shown between vitamin A status and the nephron endowment of newborns in the rodent model (11), and RA administration during midgestation has been shown to prevent the $30 \%$ nephron deficit expected because of intrauterine growth restriction in rat offspring (12).

Given the importance of RA signaling during kidney development, it is conceivable that the administration of vitamin A derivatives after preterm birth may act to augment ongoing nephrogenesis. In this regard, recommendations for vitamin A supplementation in neonates are currently in place (13), as it has previously been demonstrated that low birth weight and preterm neonates have low levels of both hepatic and serum retinol $(14,15)$. A Cochrane Review of clinical trials has since established that retinol supplementation in low birth weight infants results in improved vitamin A status leading to a reduction in neonatal mortality and oxygen requirement at 1 mo of age (16). This may be due to a beneficial effect of vitamin A supplementation on lung development, as has been evidenced in a number of experimental studies (17-20).

Abbreviation: RA, Retinoic acid 
The effects of postnatal vitamin A supplementation on kidney development, however, are currently unknown. The major aim of this study, therefore, was to determine whether early postnatal administration of RA could stimulate nephrogenesis in a nonhuman primate model of preterm birth. Fetal baboons were delivered at $125 \mathrm{~d}$ gestation $($ term $=185 \mathrm{~d}$ ), equivalent to approximately $26 \mathrm{wk}$ gestation in humans, and placed in a neonatal intensive care unit after birth (21). Importantly, the ontogeny of the baboon kidney follows very closely to that of human renal development (22), with ongoing nephrogenesis after preterm birth (Gubhaju L et al. The effects of preterm birth on nephrogenesis, 32nd Annual FNPS Meeting, September 25-28, 2005, Glenelg, Australia, Abstract O8).

\section{METHODS}

Induction of preterm delivery. All animal experiments were performed at the Southwest Foundation for Biomedical Research (San Antonio, TX), with animal handling and husbandry procedures reviewed and approved to conform to the American Association for Accreditation of Laboratory Animal Care guidelines.

At 48 and $24 \mathrm{~h}$ before delivery, pregnant baboons received $6 \mathrm{mg}$ of betamethasone (Celestone Soluspan; Schering-Plough Pharmaceuticals, Kenilworth, NJ) via intramuscular injection. Baboon neonates were delivered prematurely by caesarean section at $125 \mathrm{~d}$ gestation $($ term $=185 \mathrm{~d})$.

Postnatal care. A detailed description of the postnatal intensive care protocols, including nutritional and ventilatory management, has been previously published (21). As described, respiration, cardiac function, fluid levels, and urine output were all maintained within the normal parameters of the extremely low birth weight infant.

Medication. All preterm neonates were administered ampicillin and gentamicin for the first 7-10 d of life, at the recommended dosage for infants less than 29 wk gestation (23). Prophylactic fluconazole $(6 \mathrm{mg} / \mathrm{kg})$ was administered at 12, 96, and $168 \mathrm{~h}$ of age, then twice a week until postnatal day 21. Further doses of antibiotics were used in cases of clinically suspected infection. All neonates were treated with i.v. caffeine citrate $(20 \mathrm{mg} / \mathrm{kg})$ at 1 and $12 \mathrm{~h}$ of age, followed by $10 \mathrm{mg} / \mathrm{kg}$ daily.

In the case of significant hypotension, the stepwise administration of additional volume, dopamine, dobutamine, and hydrocortisone was undertaken. Patent ductus arteriosus was treated with indomethacin or surgical ligation.

Retinoic acid treatment. Baboon neonates were randomly assigned to treatment groups before delivery. The animals in the preterm +RA treatment group $(n=6)$ received $500 \mu \mathrm{g} / \mathrm{kg} / \mathrm{d}$ of all-trans $\mathrm{RA}(17-19)$ in almond oil via gavage commencing within $24 \mathrm{~h}$ of delivery. Retinol (2000 IU/kg; Aquasol A) was also administered via intramuscular injection every $3 \mathrm{~d}$ to maintain adequate serum retinol levels $(>20 \mu \mathrm{g} / \mathrm{mL})$. The administration of exogenous RA is known to have a sparing effect on hepatic retinol stores (24), leading to decreased serum retinol levels. Retinol deficiency in these animals was thereby prevented by retinol supplementation. The animals in the preterm control group were matched for both gestational and postnatal age, as well as maternal betamethasone treatment. The majority received daily vehicle injections $(n=4)$, and the remainder $(n=2)$ were untreated. Serum retinol concentrations were measured at birth, then at 7, 14, and $21 \mathrm{~d}$ postnatally.

Animal euthanasia and tissue processing. At postnatal day 21, baboons were euthanized. Body weights at birth and at the time of necropsy were recorded. Kidneys were cleaned of fat and connective tissue, cut into quarters, and immersion fixed in $10 \%$ buffered formalin. Researchers were blinded to the treatment group of each kidney during analysis.

Fixed kidney quarters were initially weighed then sliced into $2 \mathrm{~mm}$ slices using a razor blade device. Every second slice was then embedded in glycolmethacrylate to be used in the estimation of kidney volume and nephron number.

Estimation of kidney volume, renal corpuscle volume, and nephron number. Glycolmethacrylate blocks were serially sectioned at $20 \mu \mathrm{m}$ with every 10th and 11th section collected and stained with hemotoxylin and eosin. Kidney volume was then estimated using the Cavalieri principle (26).

In each kidney, the number of glomeruli (thereby the number of nephrons) and renal corpuscle volume were estimated using an unbiased physical disector/fractionator technique $(27,28)$.

Determination of glomerular generation number. Medullary ray glomerular generations are the number of glomeruli formed in succession from the

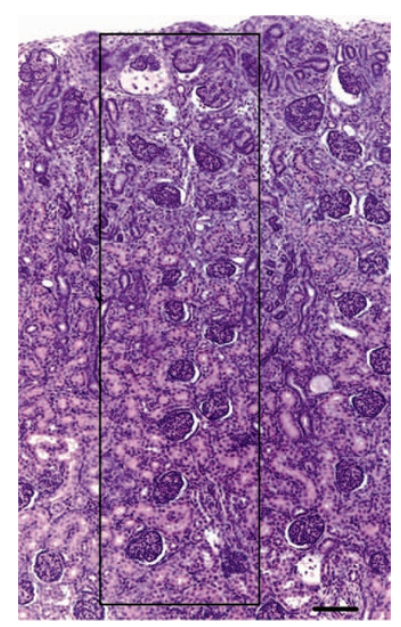

Figure 1. Photomicrograph of a neonatal baboon kidney indicating the glomerular generation counting method. Mature glomeruli were counted in a straight line along one side of a clearly distinguishable medullary ray (indicated by the black box) from the corticomedullary junction to the outer renal cortex. Scale bar $=100 \mu \mathrm{m}$.

corticomedullary junction (first to be formed) to the outer renal cortex (last to be formed). One complete section from every kidney block was used to count the number of glomeruli per ray (Fig. 1), in five clearly distinguishable medullary rays per section (approximately 40 rays per kidney). An average number of glomerular generations for each kidney were then determined. This method is considered to be highly reproducible (29) and has previously been used in the study of nephrogenesis in preterm human neonates $(5,30)$.

Determination of the percentage of abnormal glomeruli. A number of glomeruli situated in the outer renal cortex of several kidney sections were of abnormal appearance, with a dilated Bowman's space and shrunken glomerular tuft. In every 10th section, previously used in the estimation of nephron number, the number of normal and abnormal glomeruli was recorded in each of the sampled fields of view. The percentage of abnormal glomeruli was then calculated.

Immunohistochemical localization of the endothelial cell marker, CD31 and the podocyte marker, wilms tumor suppressor gene 1 (WT-1). Fourmicrometer paraffin sections, from both the preterm control and preterm +RA animals, were deparaffinized and rehydrated, then underwent heat-induced antigen retrieval in sodium citrate buffer $(10 \mathrm{mM}$ sodium citrate, pH6.0) for CD31 localization, and tris-EDTA buffer $(10 \mathrm{mM}$ Tris Base, $1 \mathrm{mM}$ EDTA, $0.05 \%$ Tween 20, $\mathrm{pH} 9.0$ ) for the localization of WT-1. Endogenous peroxidase activity was blocked for $15 \mathrm{~min}$ with endogenous enzyme block solution (Dako, CA) before incubation with $1 \%$ goat serum for $30 \mathrm{~min}$. Sections were incubated overnight at room temperature with a monoclonal mouse antihuman WT-1 at 1:100 dilution, or CD31 antibody (Dako, CA). The sections were subsequently incubated for $1 \mathrm{~h}$ with the "Envision" molecule (Dako, CA), and $3^{\prime} 3^{\prime}$-diaminobenzidine tetrachloride was used to detect antibody binding. All sections were counterstained with hematoxylin.

Synthesis of riboprobes. A cDNA fragment of human $\mathrm{VEGF}_{121}$ (gift of Steven Stacker; Ludwig Institute, Melbourne, Australia) was cloned into BSKS plasmid (Stratagene, CA) and linearized with HindIII. An antisense riboprobe was generated from the template incorporating digoxigenin (DIG)UTP (Roche Applied Science, Mannheim, Germany) into run off transcripts using T7 RNA polymerase. A sense riboprobe was also generated.

In situ hybridization. In situ hybridization of VEGF was performed on 4 $\mu \mathrm{m}$ paraffin sections from both preterm control and preterm $+\mathrm{RA}$ animals. Paraffin sections were deparaffinized and rehydrated before being microwaved in $0.01 \mathrm{M}$ citrate buffer, $\mathrm{pH} 6.0$, for $12 \mathrm{~min}$, and postfixed in $1 \%$ formaldehyde in phosphate buffered saline. The sections were incubated in proteinase $\mathrm{K}$ (Roche Applied Science, Mannheim, Germany) for $20 \mathrm{~min}$ at $37^{\circ} \mathrm{C}$, then prehybridized for $1 \mathrm{~h}$ in hybridization solution [50\% deionized formamide, $5 \times \mathrm{SSC}, 0.02 \%$ SDS, $0.1 \% \mathrm{~N}$-laurosarcosine, DEPC $\mathrm{H}_{2} \mathrm{O}, 10 \%$ blocking reagent (Roche Applied Science, Mannheim, Germany)]. Sections were then hybridized overnight at $50^{\circ} \mathrm{C}$ with DIG-labeled VEGF antisense and sense riboprobes at $1 / 10$ dilution in hybridization buffer. Slides were then washed in $2 \times$ SSC, and treated with RNAse $(150 \mu \mathrm{g} / \mathrm{mL}$ in $2 \times \mathrm{SSC})$ for $30 \mathrm{~min}$ at $37^{\circ} \mathrm{C}$. After RNAse treatment, slides were washed in $1 \times \mathrm{SSC}, 0.1 \times \mathrm{SSC}$, then maleic acid buffer ( $0.1 \mathrm{M}$ maleic acid, $0.15 \mathrm{M} \mathrm{NaCl}$; $\mathrm{pH} 7.5$ ) for $5 \mathrm{~min}$ at room temperature. Sections were incubated in $10 \%$ blocking reagent (Boehringer, Mannheim, Germany) for $30 \mathrm{~min}$, followed by $60 \mathrm{~min}$ incuba- 
tion with 1/500 anti-DIG-AP, Fab fragments (Roche Applied Science, Mannheim, Germany) in blocking reagent. Sections were incubated overnight at room temperature with BCIP/NBT chromagen substrate (DakoCytomation, CA), then coverslipped using Clearmount mounting medium (Zymed, CA).

Statistical analysis. Statistical analyses were performed using GraphPad Prism Version 4.03 for Windows (GraphPad Software, CA). An unpaired $t$ test was used to compare data from the preterm control and preterm $+\mathrm{RA}$ groups. Linear regression analyses were performed to determine whether there were any significant correlations between birth/necropsy weight and kidney weight, nephron number and kidney wt/vol and renal corpuscle volume. An analysis of covariance (ANCOVA) was also performed to determine whether there were any significant differences in the linear regression of nephron number versus kidney volume and kidney weight between the preterm control and preterm + RA groups. Statistical significance was accepted as $p<0.05$

\section{RESULTS}

Birth weight, necropsy weight, and kidney weight. There was no significant difference between the preterm control and preterm + RA groups in body weights at birth or at the time of necropsy. There was also no significant difference in the weights of the fixed kidneys (Table 1).

Linear regression analyses revealed a significant correlation between birth weight and kidney weight in the preterm control group $\left(R^{2}=0.771, p=0.02\right)$. This association, however, was not evident in the preterm +RA group $\left(R^{2}=0.342, p=0.22\right)$. Similarly, there was no significant correlation between necropsy weight and kidney weight in the preterm +RA group $\left(R^{2}=0.080, p=0.59\right)$. The association was nearing significance, however, in the preterm controls $\left(R^{2}=0.627\right.$, $p=0.06)$.

Clinical measurements. There were no statistically significant differences between the preterm control and preterm $+\mathrm{RA}$ animals in arterial blood gas pressure $\left(\mathrm{Po}_{2}, \mathrm{PCO}_{2}\right)$, blood pressure, fluid intake, or urine output at postnatal day 21 (Table 2).

The serum retinol concentration of the preterm control $(n=3)$ and preterm + RA $(n=6)$ neonatal baboons did not differ significantly at birth, $7 \mathrm{~d}$, or $21 \mathrm{~d}$. There was, however, a significantly higher mean serum retinol concentration in animals in the preterm control group at postnatal day 14 (preterm control: $45.733 \pm 5.385 \mathrm{mg} / 100 \mathrm{~mL}$; preterm +RA: $23.700 \pm$ $3.071 \mathrm{mg} / 100 \mathrm{~mL} ; p=0.01)$.

Kidney volume and renal corpuscle volume. Kidney volumes and renal corpuscle volumes are shown in Table 1. There was no significant difference between the two treatment groups in either parameter.

Table 1. Mean birth and necropsy weights, kidney weights and volumes, renal corpuscle volumes, and nephron density data of the preterm control and preterm $+R A$ groups

\begin{tabular}{lcc}
\hline & $\begin{array}{c}\text { Preterm control } \\
(n=6)\end{array}$ & $\begin{array}{c}\text { Preterm +RA } \\
(n=6)\end{array}$ \\
\hline Birth weight $(\mathrm{g})$ & $391.8 \pm 20.6$ & $382.7 \pm 16.7$ \\
Necropsy weight $(\mathrm{g})$ & $372.8 \pm 24.8$ & $383.3 \pm 27.1$ \\
Kidney weight $(\mathrm{g})$ & $1.85 \pm 0.26$ & $2.07 \pm 0.30$ \\
Kidney volume $\left(\mathrm{mm}^{3}\right)$ & $1156 \pm 164$ & $1229 \pm 178$ \\
Renal corpuscle volume $\left(\times 10^{-4} \mathrm{~mm}^{3}\right)$ & $2.60 \pm 0.23$ & $2.42 \pm 0.17$ \\
Nephron density $\left(\mathrm{glomeruli} / \mathrm{mm}^{3}\right)$ & $298.8 \pm 28.2$ & $322.6 \pm 23.6$ \\
\hline
\end{tabular}

Data are expressed as the mean \pm SEM. There was no significant difference between groups in any parameter.
Table 2. Measurements of blood pressure, arterial blood gas pressures, fluid intake and urine output in preterm control and preterm $+R A$ animals at postnatal day 21

\begin{tabular}{lcc}
\hline & $\begin{array}{c}\text { Preterm control } \\
(n=6)\end{array}$ & $\begin{array}{c}\text { Preterm +RA } \\
(n=6)\end{array}$ \\
\hline Systolic blood pressure $(\mathrm{mm} \mathrm{Hg})$ & $53 \pm 4$ & $56 \pm 13$ \\
Diastolic blood pressure $(\mathrm{mm} \mathrm{Hg})$ & $21 \pm 4$ & $25 \pm 6$ \\
Mean blood pressure $(\mathrm{mm} \mathrm{Hg})$ & $33 \pm 2$ & $35 \pm 8$ \\
$\mathrm{Po}_{2}(\mathrm{~mm} \mathrm{Hg})$ & $67.0 \pm 5.1$ & $77.2 \pm 11.7$ \\
$\mathrm{PCO}_{2}(\mathrm{~mm} \mathrm{Hg})$ & $51.2 \pm 1.0$ & $44.2 \pm 3.7$ \\
Fluid intake $(\mathrm{mL} / \mathrm{kg} / \mathrm{d})$ & $167.8 \pm 5.2$ & $181.4 \pm 5.6$ \\
Urine output $(\mathrm{mL} / \mathrm{kg} / \mathrm{h})$ & $3.1 \pm 0.5$ & $2.5 \pm 0.8$ \\
\hline
\end{tabular}

Data are expressed as the mean \pm SEM. There was no significant difference between groups in any parameter.

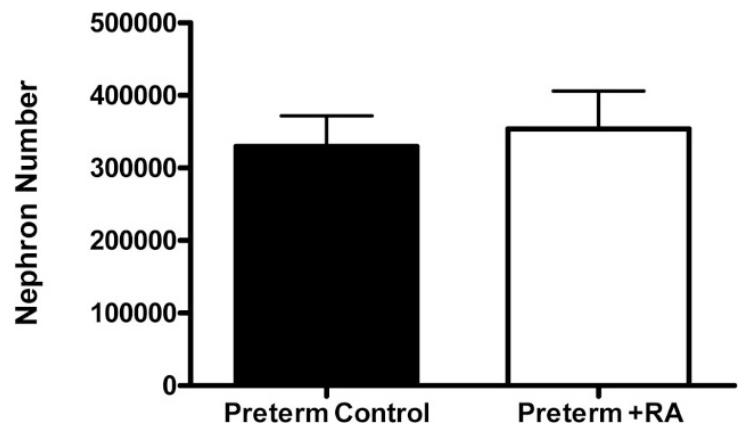

Figure 2. Bar graph depicting the average nephron number $( \pm$ SEM) of preterm control $(n=6)$ and preterm + RA $(n=6)$ animals.

Nephron number and density. Average nephron number for the preterm control and preterm +RA groups is shown in Figure 2. There was no significant difference between the two treatment groups. Similarly, there were no significant differences in nephron density (number of glomeruli per unit volume of kidney), as shown in Table 1.

There was a significant correlation between kidney volume and nephron number within both the preterm control and preterm + RA groups, as depicted in Figure 3. Furthermore, a comparison between the two regression lines (ANCOVA) revealed no significant difference between the slope values, and predicted an overall increase of 236 nephrons per $1 \mathrm{~mm}^{3}$ increase in kidney volume across the pooled data.

Correlations between kidney weight and nephron number within the preterm control $\left(R^{2}=0.613, p=0.07\right)$ and the preterm $+\mathrm{RA}\left(R^{2}=0.611, p=0.07\right)$ treatment groups did not quite reach statistical significance. Because an ANCOVA analysis revealed no significant difference between the

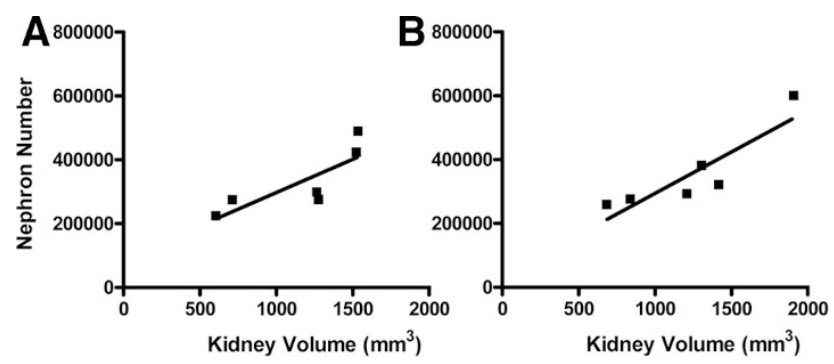

Figure 3. Linear regression analyses of nephron number $v s$ kidney volume in $A$ preterm control $\left(R^{2}=0.665, p=0.047\right)$, and $B$ preterm $+\mathrm{RA}\left(R^{2}=0.785\right.$, $p=0.019)$ animals. 

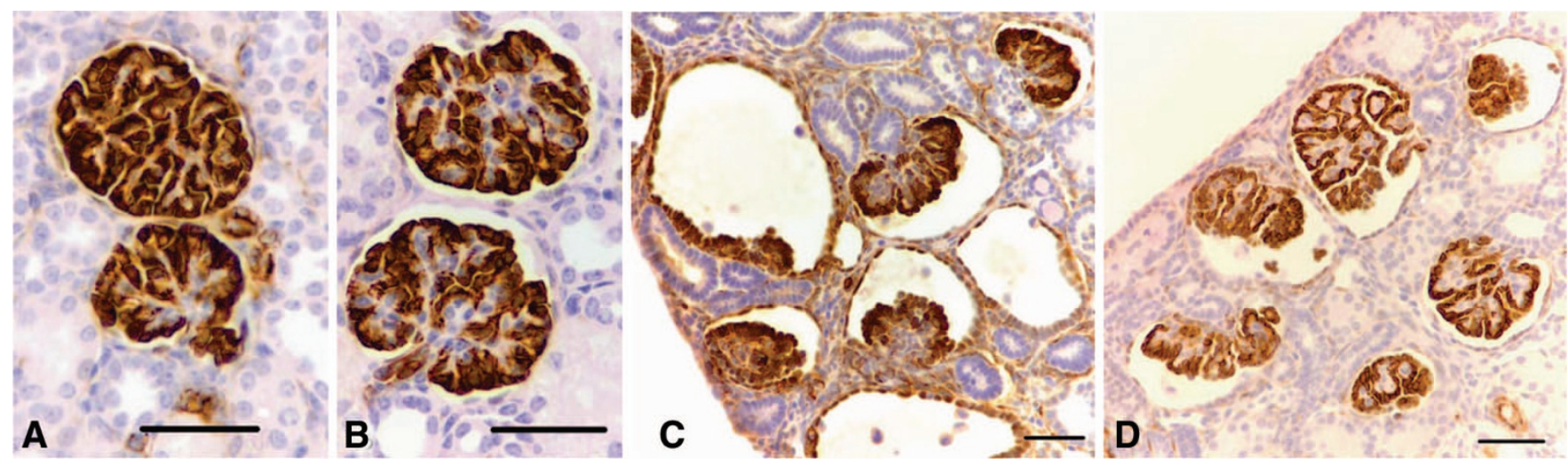

Figure 4. Representative photomicrographs of WT-1 immunostaining of podocytes in normal and abnormal glomeruli from both preterm control ( $A$, normal; $C$, abnormal) and preterm $+\mathrm{RA}$ animals $(B$, normal; $D$, abnormal). Scale bar $=50 \mu \mathrm{m}$.
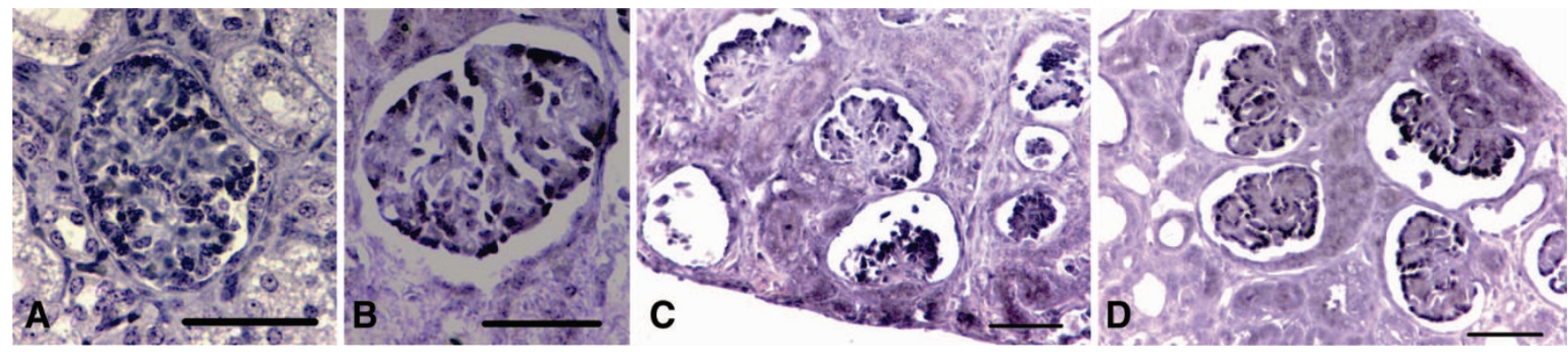

Figure 5. Representative photomicrographs of in situ hybridization of VEGF expression (dark purple stained nuclei) in podocytes of normal and abnormal glomeruli from preterm control $(A$, normal; $C$, abnormal) and preterm + RA animals $(B$, normal; $D$, abnormal). Scale bar $=50 \mu \mathrm{m}$.

slope values of the two linear regressions, data from the two treatment groups were pooled and subsequently revealed a highly significant correlation between nephron number and kidney weight $\left(R^{2}=0.672, p=0.001\right)$ across all preterm animals.

Further linear regression analyses revealed no significant correlations between body weight and nephron number or nephron number and renal corpuscle volume.

Glomerular generations. There was no significant difference in the number of glomerular generations formed within the kidneys of the preterm control or preterm + RA animals (preterm control: $10.04 \pm 0.26$; preterm + RA: $9.99 \pm 0.48$; $p=0.91)$. The average number of glomerular generations per medullary ray was similar in all kidneys studied.

Abnormal glomeruli. Abnormal glomeruli were classified as those with cystic dilation of the Bowman's space. These glomeruli also commonly exhibited a shrunken glomerular tuft, as depicted in Figures 4-6 $(C$ and $D)$. Importantly, the abnormal glomeruli appeared only in the outer renal cortex of the kidney, whereas glomeruli located in the inner cortex of the same kidneys appeared to be normal.

There was a wide range in the proportion of abnormal glomeruli in the kidneys of the preterm control (0.7-13.9\%) and the preterm +RA $(0.0-16.0 \%)$ animals. There was no significant difference in the proportion of abnormal glomeruli between the two treatment groups (preterm control: $3.54 \pm$ $2.10 \%$; preterm + RA: $5.83 \pm 2.62 \% ; p=0.51)$. As shown in Table 3, there was no clear link between the administration of any of the postnatal medications and the percentage of abnormal glomeruli.

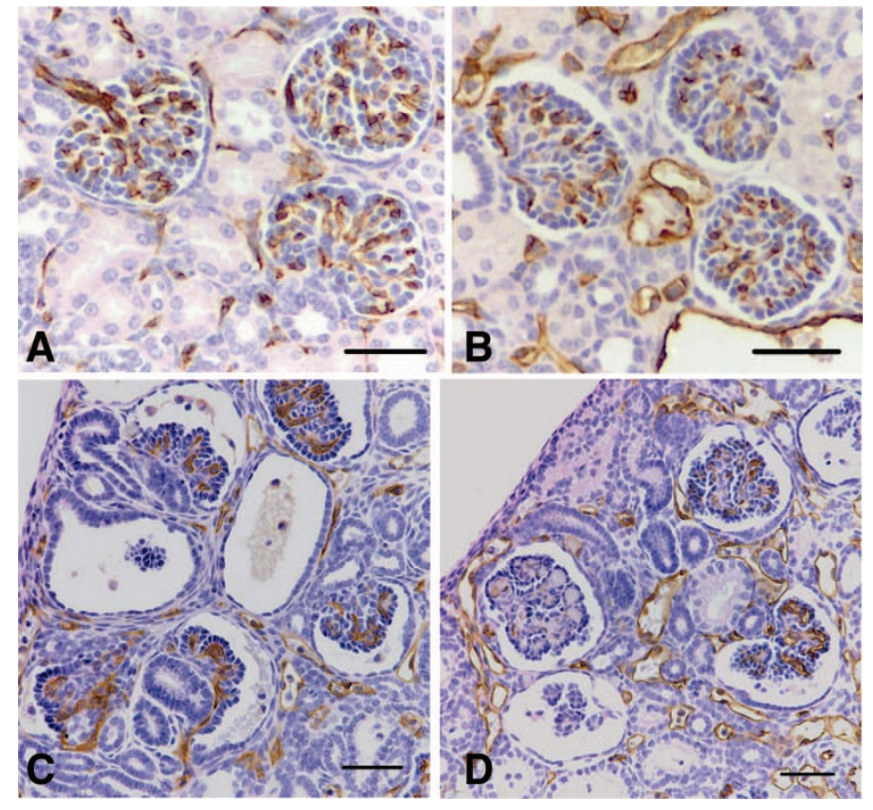

Figure 6. Representative photomicrographs of CD31 immunostaining of endothelial cells in normal and abnormal glomeruli from preterm control $(A$, normal; $C$, abnormal) and preterm + RA animals $(B$, normal; $D$, abnormal). Scale bar $=50 \mu \mathrm{m}$.

\section{Immunohistochemical Localization and In Situ Hybridization}

Podocyte marker WT-1. WT-1 immunostaining was localized to visceral and parietal podocytes in both normal and abnormal glomeruli from the preterm control and preterm + RA 
Table 3. Medications administered postnatally to individual preterm control and preterm + RA animals, when compared with the percentage of abnormal glomeruli

\begin{tabular}{|c|c|c|c|c|c|c|c|c|c|c|c|c|c|}
\hline Group & $\%$ Abnormal & Ampicillin & $\begin{array}{l}\text { Genta- } \\
\text { micin }\end{array}$ & $\begin{array}{l}\text { Vanco- } \\
\text { mycin }\end{array}$ & Fortaz & Indomethacin & Caffeine & Dopamine & Dobutamine & $\begin{array}{l}\text { Hydro- } \\
\text { cortisone }\end{array}$ & $\begin{array}{l}\text { Fluco- } \\
\text { nazole }\end{array}$ & Cancidas & Nystatin \\
\hline $\begin{array}{l}\text { Preterm } \\
\text { control }\end{array}$ & $\begin{array}{r}13.93 \\
1.83 \\
1.04 \\
1.14 \\
0.73 \\
2.56\end{array}$ & $\begin{array}{l}V \\
V \\
V \\
V \\
V \\
V\end{array}$ & $\begin{array}{l}V \\
V \\
V \\
V \\
V \\
V\end{array}$ & V & V & V & $\begin{array}{l}V \\
V \\
V \\
V \\
V \\
V\end{array}$ & $\begin{array}{l}V \\
V \\
V \\
V \\
V\end{array}$ & V & V & $\begin{array}{l}V \\
V \\
V \\
V \\
V \\
V\end{array}$ & & \\
\hline $\begin{array}{r}\text { Preterm } \\
+ \text { RA }\end{array}$ & $\begin{array}{r}4.12 \\
11.29 \\
16.03 \\
0.55 \\
0.00 \\
2.99\end{array}$ & $\begin{array}{l}V \\
V \\
V \\
V \\
V \\
V\end{array}$ & $\begin{array}{l}V \\
V \\
V \\
V \\
V \\
V\end{array}$ & V & & V & $\begin{array}{l}V \\
V \\
V \\
V \\
V \\
V\end{array}$ & . & & & $\begin{array}{l}V \\
V \\
V \\
V \\
V \\
V\end{array}$ & V & $\begin{array}{l}\text { V } \\
\text { V }\end{array}$ \\
\hline
\end{tabular}

animals (Fig. 4). In the abnormal glomeruli, podocytes were localized to a crescent of cells lining the glomerular tuft, indicating that the glomeruli are at an immature stage of development (31). Glomeruli that were grossly abnormal exhibited a glomerular tuft that was composed primarily of podocytes.

Vascular endothelial growth factor. In situ hybridization of VEGF mRNA indicated that VEGF was expressed by the podocytes in all normal and abnormal glomeruli from the preterm control and preterm +RA animals (Fig. 5).

Endothelial cell marker CD31. CD31 immunostaining was localized to the capillaries within the glomerular tuft of normal and abnormal glomeruli, in both the preterm control and preterm +RA animals (Fig. 6). Glomeruli that were grossly abnormal lacked CD31 immunostaining indicating that they were not capillarized.

\section{DISCUSSION}

The results of this study indicate that early postnatal administration of RA does not stimulate nephrogenesis in the kidney of the preterm baboon. Furthermore, RA treatment was shown to have no influence on postnatal growth or kidney size. It was observed, however, that a number of glomeruli located in the outer renal cortex of the preterm baboon kidney were of abnormal appearance, suggesting that nephrons formed in the extrauterine environment are at risk of impairment.

Contrary to our initial hypothesis, the results demonstrate that daily RA administration $(500 \mu \mathrm{g} / \mathrm{kg} / \mathrm{d})$ has no significant effect on nephron number, the number of glomerular generations, kidney volume and weight, renal corpuscle volume, and the proportion of abnormal glomeruli formed. The lack of effect of RA administration on nephrogenesis in the present study may relate to the timing of administration. RA administered during mid-gestation has been found to have a strong influence on branching morphogenesis in kidney development $(11,12)$, as it was administered at a time when the ureteric bud was undergoing its initial levels of branching. Conversely, in the present study, RA was administered to the prematurely delivered baboons at a time point consistent with $26 \mathrm{wk}$ of gestation onwards, where up to 10 generations of glomeruli have already formed. In the human kidney, branching occurs only very rarely after the $22 \mathrm{nd}$ wk of gestation (32) as the ampullae of the ureteric buds seldom divide and new nephrons are formed in an arcade pattern, attached to single ampullae $(3,32)$. Hence, nephrogenesis in the postnatal preterm kidney may not have been receptive to the influence of RA at this time. Taken together with findings from previous studies, the results suggest than an adequate maternal supply of vitamin A during gestation is of more importance in achieving optimal nephron endowment after premature birth than is the influence of postnatal supplementation.

In the current study, there was a significant linear correlation between nephron number and both kidney weight and kidney volume across all preterm baboons, as has been shown previously (22). Therefore, the total number of nephrons formed in these animals is strongly linked to individual kidney size. In this regard, kidney size was not influenced by RA treatment and nor was nephron number. Nephron number in the preterm baboon kidney ranged between 273,697 and 599,320 , which is consistent with previously published findings in the baboon (22), albeit in the higher range. Furthermore, the number of glomerular generations was found to be very similar across all animals, with approximately 10 generations of glomeruli formed in the prematurely delivered baboon kidney by postnatal day 21 of development.

Although a nephron deficit as such has not been exhibited after preterm birth, a reduction in the functionality of the kidney is likely. In the current study, it was noted that a number of glomeruli exhibiting a dilated Bowman's space and shrunken glomerular tuft were present in the outer renal cortex of kidneys from both the preterm groups. The overall proportion of abnormal glomeruli ranged from 0 to $16 \%$, and these were seen to be situated exclusively in the outer renal cortex of the kidney, suggesting that only those glomeruli formed in the extrauterine environment are at risk of impairment. In support of this concept, our laboratory has recently shown that the number of abnormal glomeruli in the kidneys of term and gestational control animals is negligible (Gubhaju L et al. The effects of preterm birth on nephrogenesis, 32nd Annual FNPS Meeting, September 25-28, 2005, Glenelg, Australia, Abstract O8), indicating that the glomerular abnormalities have occurred as a direct result of premature birth and/or its associated complications. Through immunohistochemical and in situ 
hybridization analysis, we have determined that VEGF, the major angiogenic growth factor for glomerular capillaries, is expressed by the podocytes of the abnormal glomeruli and there is some evidence of capillarization of the tuft. In the grossly abnormal glomeruli, however, endothelial cell staining was negative suggesting that they are nonfunctional.

However, the cause of the abnormal glomerular morphology is, at the present time, unknown. In this regard, there was no link between the proportion of abnormal glomeruli in the preterm kidney and any irregularities in clinical parameters, such as $\mathrm{PO}_{2}$ and $\mathrm{PCO}_{2}$ levels. Furthermore, there was no apparent link between the percentage of abnormal glomeruli and medications administered postnatally (Table 3). Although medications such as aminoglycoside antibiotics and nonsteroidal antiinflammatory drugs have been reported to lead to renal injury in experimental studies (33-35) and impaired renal function in human neonates (36), there is no evidence to suggest that this was the cause of the abnormal glomeruli in the current study. All animals were routinely administered gentamicin for the first 7-10 d of life, and three animals received indomethacin; however, only one of these three neonates had a high proportion of abnormal glomeruli.

In conclusion, the results of this study indicate that early postnatal administration of RA is unable to enhance nephron endowment in the kidney of the preterm baboon, and this lack of effect is likely to be due to the timing of administration. As vitamin A supplementation is already being trialed in humans, however, it was encouraging to note that postnatal administration of RA and retinol does not appear to have an adverse effect on kidney development.

Acknowledgments. The authors thank the Southwest Foundation for Biomedical Research (San Antonio, TX, USA) and in particular Professor Jacqueline Coalson and Ms. Vicki Winter from the University of Texas Health Science Center for their assistance in obtaining the baboon kidneys. We also thank Associate Professor Darren Kelly and Ms. Alison Cox, from the Department of Medicine, St. Vincent's Hospital, Melbourne, for undertaking the in situ hybridization.

\section{REFERENCES}

1. Noble L 2003 Developments in neonatal technology continue to improve infant outcomes. Pediatr Ann 32:595-603

2. Slattery MM, Morrison JJ 2002 Preterm delivery. Lancet 360:1489-1497

3. Saxen L 1987 Organogenesis of the Kidney. Cambridge: Cambridge University Press

4. Hinchliffe SA, Sargent PH, Howard CV, Chan YF, Van Velzen D 1991 Human intrauterine renal growth expressed in absolute number of glomeruli assessed by the disector method and cavalieri principle. Lab Invest 64:777-784

5. Rodriguez MM, Gomez AH, Abitbol CL, Chandar JJ, Duara S, Zilleruelo GE 2004 Histomorphometric analysis of postnatal glomerulogenesis in extremely preterm infants. Pediatr Dev Pathol 7:17-25
6. Hoy WE, Hughson MD, Bertram JF, Douglas-Denton R, Amann K 2005 Nephron number, hypertension, renal disease, and renal failure. J Am Soc Nephrol 16:25572564

7. Brenner BM, Mackenzie HS 1997 Nephron mass as a risk factor for progression of renal disease. Kidney Int Suppl 63:S124-S127

8. Nenov VD, Taal MW, Sakharova OV, Brenner BM 2000 Multi-hit nature of chronic renal disease. Curr Opin Nephrol Hypertens 9:85-97

9. Moreau E, Vilar J, Lelievre-Pegorier M, Merlet-Benichou C, Gilbert T 1998 Regulation of c-ret expression by retinoic acid in rat metanephros: implication in nephron mass control. Am J Physiol 275:F938-F945

10. Vilar J, Gilbert T, Moreau E, Merlet-Benichou C 1996 Metanephros organogenesis is highly stimulated by vitamin A derivatives in organ culture. Kidney Int 49:1478-1487

11. Lelievre-Pegorier M, Vilar J, Ferrier M-L, Moreau E, Freund N, Gilbert T, MerletBenichou C 1998 Mild vitamin A deficiency leads to inborn nephron deficit in the rat. Kidney Int 54:1455-1462

12. Makrakis J, Zimanyi MA, Black MJ 2007 Retinoic acid enhances nephron endowment in rats exposed to maternal protein restriction. Pediatr Nephrol 22:1861-1867

13. Kennedy KA, Stoll BJ, Ehrenkranz RA, Oh W, Wright LL, Stevenson DK, Lemons JA, Sowell A, Mele L, Tyson JE, Verter J 1997 Vitamin A to prevent bronchopulmonary dysplasia in very-low-birth-weight infants: has the dose been too low? The NICHD Neonatal Research Network. Early Hum Dev 49:19-31

14. Shenai JP, Chytil F, Jhaveri A, Stahlman MT 1981 Plasma vitamin A and retinolbinding protein in premature and term neonates. J Pediatr 99:302-305

15. Shenai JP, Chytil F, Stahlman MT 1985 Liver vitamin A reserves of very low birth weight neonates. Pediatr Res 19:892-893

16. Darlow BA, Graham PJ 2007 Vitamin A supplementation to prevent mortality and short and long-term morbidity in very low birthweight infants. Cochrane Database Syst Rev 4:CD000501

17. Massaro GD, Massaro D 1996 Postnatal treatment with retinoic acid increases the number of pulmonary alveoli in rats. Am J Physiol 270:L305-L310

18. Massaro GD, Massaro D 1997 Retinoic acid treatment abrogates elastase-induced pulmonary emphysema in rats. Nat Med 3:675-677

19. Massaro GD, Massaro D 2000 Retinoic acid treatment partially rescues failed septation in rats and in mice. Am J Physiol Lung Cell Mol Physiol 278:L955-L960

20. Veness-Meehan KA, Pierce RA, Moats-Staats BM, Stiles AD 2002 Retinoic acid attenuates O2-induced inhibition of lung septation. Am J Physiol Lung Cell Mol Physiol 283:L971-L980

21. Thomson MA, Yoder BA, Winter VT, Martin H, Catland D, Siler-Khodr TM, Coalson JJ 2004 Treatment of immature baboons for 28 days with early nasal continuous positive airway pressure. Am J Respir Crit Care Med 169:1054-1062

22. Gubhaju L, Black MJ 2005 The baboon as a good model for studies of human kidney development. Pediatr Res 58:505-509

23. Young TE, Mangum OB 2002 Neofax. A manual of drugs used in neonatal care. Raleigh, NC: Acorn Publishing

24. Gerlach TH, Zile MH 1991 Effect of retinoic acid and apo-RBP on serum retinol concentration in acute renal failure. FASEB J 5:86-92

25. Deleted in proof

26. Gundersen HJ, Jensen EB 1987 The efficiency of systematic sampling in stereology and its prediction. J Microsc 147:229-263

27. Bertram JF 2001 Counting in the kidney. Kidney Int 59:792-796

28. Black MJ, Briscoe TA, Constantinou M, Kett MM, Bertram JF 2004 Is there an association between level of adult blood pressure and nephron number or renal filtration surface area? Kidney Int 65:582-588

29. Hinchliffe SA, Sargent PH, Chan YF, van Velzen D, Howard CV, Hutton JL, Rushton DI 1992 "Medullary ray glomerular counting" as a method of assessment of human nephrogenesis. Pathol Res Pract 188:775-782

30. Rodriguez MM, Gomez A, Abitbol C, Chandar J, Montane B, Zilleruelo G 2005 Comparative renal histomorphometry: a case study of oligonephropathy of prematurity. Pediatr Nephrol 20:945-949

31. Vernier RL, Birch-Andersen A 1962 Studies of the human fetal kidney. I. Development of the glomerulus. J Pediatr 60:754-768

32. Osathanondh V, Potter E 1963 Development of human kidney as shown by microdissection. III. Formation and interrelationship of collecting tubules and nephrons. Arch Pathol 76:290-302

33. Kent AL, Maxwell LE, Koina ME, Falk MC, Willenborg D, Dahlstrom JE 2007 Renal glomeruli and tubular injury following indomethacin, ibuprofen, and gentamicin exposure in a neonatal rat model. Pediatr Res 62:307-312

34. Gilbert T, Gaonach S, Moreau E, Merlet-Benichou C 1994 Defect of nephrogenesis induced by gentamicin in rat metanephric organ culture. Lab Invest 70:656-666

35. Cullen LA, Young RJ, Bertram JF 2000 Studies on the effects of gentamicin on rat metanephric development in vitro. Nephrology 5:115-123

36. Andreoli SP 2004 Acute renal failure in the newborn. Semin Perinatol 28:112-123 